\title{
Performance evaluation of parallel TCP, and its impact on bandwidth utilization and fairness in high-BDP networks based on test-bed
}

\begin{abstract}
After the presence of high Bandwidth-Delay Product (high-BDP) networks, many researches have been conducted to prove either the existing TCP variants can achieve an excellent performance without wasting the bandwidth of these networks or not. In this paper, a comparative test-bed experiment on a set of high speed TCP variants has been conducted to show their differences in bandwidth utilization, loss ratio and TCP-Fairness. The involved TCP Variants in this experiment are: NewReno, STCP, HS-TCP, H-TCP and CUBIC. These TCP variants have been examined in both cases of single and parallel schemes. The core of this work is how to evaluate these TCP variants over a single bottleneck network using a new parallel scheme to fully utilize the bandwidth of this network, and to show the impact of accelerating these variants on bandwidth utilization, loss-ratio and fairness. The results of this work reveal that, first: the proposed parallel scheme strongly outperforms the single based TCP in terms of bandwidth utilization and fairness. Second: CUBIC achieved better performance than NewReno, STCP, H-TCP and HS-TCP in both cases of single and parallel schemes. Briefly, parallel TCP scheme increases the utilization of network resources, and it is relatively good in fairness.
\end{abstract}

Keyword: Parallel TCP; Bandwidth utilization; TCP fairness; High-BDP networks 